\title{
Parsing brain activity with fMRI and mixed designs: What kind of a state is neuroimaging in?
}

\author{
Neuroimaging is often pilloried for being little more than pretty pictures that simply \\ show where activity occurs in the brain. Strong critics (notably Uttal, 2001) have \\ even argued that neuroimaging is nothing more than a modern day version of \\ phrenology; destined to fail, and fundamentally uninformative. Here I make the \\ opposite case, arguing that neuroimaging is in a vibrant and healthy state of \\ development. As recent investigations of memory illustrate, when used well, \\ neuroimaging goes beyond asking 'where' activity is occurring, to ask questions \\ concerned more with 'what' functional role that activity reflects.
}

Over the last couple of years it seems that mention of neuroimaging, be it in formal talks or informal discussion, leads many scientists to shake their heads and mutter disparagingly. The dominant method, fMRI (functional Magnetic Resonance Imaging), often receives particular disdain for offering nothing more than fancy colour pictures of the brain. The typical refrain can be paraphrased as 'Who cares which bits of the brain light up during your semantic priming task (or social attention task, or error detection task)?' And to some degree this is an understandable complaint. Indeed, if that is all fMRI offers, is it really useful knowledge? Does it deserve the financial investment and research time which have been spent on it? Fundamentally, one has to ask if knowing just 'where it is in the brain' is actually worth knowing.

As a psychologist who uses neuroimaging methods I would have sympathy with this critique - if it were true. However, I am writing this article, and continue to do neuroimaging, because I believe that the typical critique is often based on a lack of awareness of the current state (and continuing development) of the field. It would be more than fair to ridicule and resent neuroimaging if it did only answer questions about 'where' activity occurs in the brain. But the best neuroimaging studies always aim to go beyond just asking 'where', and try to answer questions about 'what' the activity reflects - why the activity is occurring. The purpose of this review is to highlight a number of recent studies that have attempted to answer 'what' questions by using an approach to neuroimaging that the disparaging critic is usually surprised to hear about, and which does not assume that answering 'where' is synonymous with answering 'what'.

Neuroimaging is for parsing, not just mapping, the brain.

A significant recent development in fMRI has been the introduction of 'mixed designs', which allow researchers to distinguish between state-related and item-related processing $(4,5,13)$. Mixed designs are a good example of how developments in the design and analysis of fMRI experiments can open up new avenues of investigation, allowing a principled distinction to be drawn between different classes of cognitive operation. Specifically, mixed designs dissociate brain regions according to the broad functional role that each region plays (not only identifying where is active) by separating brain regions based on the timecourse of their activity. The logic of mixed designs is illustrated in Fig. 1: the basic idea is that fMRI can be used to 'parse' rather than simply 'map' the 
brain. In linguistics 'parsing' refers to the assignment of constituent structure to a sentence. Without adequate organisation a sentence is potentially ambiguous or even meaningless. An analogous task is required if neuroimaging is to provide psychologically meaningful data, using experimental design to parse brain activity into it's constituent parts.

Although neuroimaging data can undoubtedly be parsed in many different ways, in the case of mixed designs the essential idea is to examine the temporal profile of activity within each region of the brain. Examining the timecourse of activity allows separation of transient (rapid or tonic) and sustained (slow or phasic) signal changes. From a psychological perspective these two patterns of signal change would be expected to reflect functional differences in what kind of role a region plays in supporting behaviour. Specifically, the distinction between transient and sustained signal changes maps onto a functional distinction between item (trial) related and state (task) related processing, and provides one clear way of characterising brain regions in functional terms - using the temporal profile of activity within each brain region to begin to outline what kind of processing the regions are doing. This is not, of course, a panacea for answering all of the what questions instantly. It does, however, allow a greater range of psychological theories to be tested, and suggests that state-related processing needs to be accounted for by these theories.

The difference between paradigm designs, and the different signals they measure, is illustrated in Figure 1. As the figure shows, 'blocked' methods (where all stimuli are presented in a block) measure all activity during a task period, averaging together (confounding) transient and sustained signals. Blocked methods simply reveal a set of 'regions of interest', regardless of differences in the underlying behaviour of these regions and their different functional roles. In contrast, 'event-related' methods (where stimuli are randomly intermixed rather than blocked) focus exclusively on extracting regions that exhibit transient changes in activity related to individual trial events, but in doing so effectively ignore any broader task-related processes that exist. By combining blocked and event-related methods into a 'mixed design' it becomes possible to independently identify and dissociate brain regions according to whether they exhibit trial- or task-related activity. In this way mixed designs effectively allows neuroimaging data to be 'parsed' in a more psychologically sophisticated fashion than is possible with either blocked or event-related methods.

\section{fMRI evidence for 'encoding mode'.}

A study by Otten et al. (9) published in Nature Neuroscience provides a particularly interesting example of a mixed design. This study follows on from a large body of neuroimaging studies that have examined memory encoding, studies that have revealed much about the functional and neural basis of the formation of memories (e.g. see 6,7,8,15, and 2,3,11 for reviews). By using a mixed design Otten et al. showed that successful memory encoding is also indexed by neural activity that is sustained (tonically maintained) throughout the performance of a study task. Subjects experienced a series of short task blocks during which they had to make semantic or phonological decisions about visually presented words as a means of encoding the words into memory. As would 
be expected on the basis of previous studies, the fMRI data revealed brain regions associated with transient activity. In addition however, the level of sustained brain activity measured across each task block was correlated with the number of words subsequently remembered from that block. The regions showing this sustained patterns of activity varied for the two different encoding tasks, with sustained activity in inferior medial parietal and left prefrontal cortex for the semantic task, and in superior medial parietal cortex for the phonological task. Of course, the interpretation of these findings depends upon appropriate theoretical models of memory encoding, but the data strongly suggest that subjects were entering a kind of 'encoding mode'. The sustained activity in these brain regions appears to support memory encoding, and models of encoding must therefore account for this state-related processing.

Otten et al. showed that a real distinction can be drawn between item- and task-related encoding processes in terms of memory encoding. Some regions exhibit sustained signal changes that support memory throughout performance of a memory task, independent of the response to the individual stimuli. This finding extends previous evidence of transient signal changes that are known to reflect the processing of individual items in memory, and suggests that accounting for encoding at the item level alone is insufficient. Importantly, this finding is not a one off. Simulation and modelling data suggests that the methodological basis for the mixed design is robust (13). Moreover, other studies of memory encoding (10), memory retrieval $(5,14)$ and task-switching $(1)$, have all used a mixed design to investigate the functional basis of cognition. In the case of memory retrieval $(5,14)$ the results provide a way to reconcile a number of disparate findings that have arisen from previous blocked and event-related studies. Thus, across a number of cognitive domains the mixed design appears to support the idea that the dynamic timecourse of processing is a critical feature of the functioning of a brain region.

\section{Caveats, assumptions, and a general principle.}

To put the development of mixed designs into some context it should be said that part of the problem for neuroimaging is that many of the most interesting questions must wait for basic knowledge to accumulate. For example, what is the relationship between neural and functional levels of analysis - does 'more' fMRI signal mean 'more' engagement of a psychological process? Similarly, what is the causal relationship between the fMRI signal and cognitive processing? Progress is required at both a theoretical and technological levels. For example, for all current methods there is a trade-off between temporal and spatial resolution, and the ideal method would not suffer this limitation. There can't be many neuroimaging researchers who believe that current methods like fMRI are the ultimate or ideal tool, even those who use it as their predominant or only method. Indeed, for the most part, researchers are aware of the limits of the tools, and are making the best use of what they have got. In this context it is clear that the development and use of mixed designs, like the development of event-related methods before it, represents a small but worthwhile improvement in the state of the art in neuroimaging research.

From the perspective of mixed designs neuroimaging is for parsing, not just mapping, the brain. Nonetheless, it is important to stress that questions of localisation are not inherently unimportant, and information about where activity is in the brain does 
contribute towards our understanding of how cognitive functions are organised in the brain. The intention here is simply to emphasis that this is not the only information provided by neuroimaging, and that information about the timecourse of processing within each region provides additional insight into function. Mixed designs provide data that is intrinsically informative about the functional behaviour of the active brain regions. In essence, I am arguing that there is more information available in neuroimaging data than the disparaging critic typically assumes. From this vantage, the neuroimaging field is in an extremely healthy state; developments such as the mixed design allow a greater range of psychological theories to be tested, and help in the attempt to answer questions about what functional role a given region plays. Such an approach still makes all of the standard assumptions upon which neuroimaging is normally based (e.g. that there is a consistent mapping between cognitive function and neuroanatomy, and that different patterns of brain activity imply different functions) and the interpretation of mixed design studies is still dependent upon theoretical accounts of cognition. However, to be adequate, theoretical accounts of cognition must take into account the functional information provided by neuroimaging data, and this now includes the general principle of a distinction between item- and state-related processing.

\section{REFERENCES:}

1. Braver TS, Reynolds JR, Donaldson DI. (2003) Neural mechanisms of transient and sustained cognitive control during task switching. Neuron, 39: 713-726.

2. Buckner RL, Logan J, Donaldson DI, Wheeler ME. (2000) Cognitive neuroscience of episodic memory encoding. Acta Psychol. Vol. 105:127-39.

3. Buckner RL, Kelley WM, Petersen SE. (1999) Frontal cortex contributes to human memory formation. Nat Neurosci, Vol. 2:311-4.

4. Donaldson DI, Buckner RL. (2001) Effective Paradigm Design. In Functional Magnetic Resonance Imaging of the Brain: Methods for Neuroscience, P.M. Matthews, P. Jezzard and A.C. Evans (Eds.) Oxford University Press.

5. Donaldson DI, Petersen SE, Ollinger JM, Buckner RL. (2001). Dissociating Item and State Components of Recognition Memory using fMRI. NeuroImage, 13: 129-142.

6. Kelley WM, Miezin FM, McDermott KB, Buckner RL, Raichle ME, Cohen NJ, Ollinger JM, Akbudak E, Conturo TE, Snyder AZ, Petersen SE. (1998) Hemispheric specialization in human dorsal frontal cortex and medial temporal lobe for verbal and nonverbal memory encoding. Neuron, Vol. 20:927-36.

7. McDermott KB, Buckner RL, Petersen SE, Kelley WM, Sanders AL. (1999) Set- and code-specific activation in frontal cortex: an fMRI study of encoding and retrieval of faces and words. J Cogn Neurosci, Vol. 11(6):631-40.

8. Otten LJ, Rugg MD. (2001) Task-dependency of the neural correlates of episodic encoding as measured by fMRI. Cereb Cortex, Vol. 11:1150-60.

9. Otten LJ, Henson RN, Rugg MD. (2002) State-related and item-related neural correlates of successful memory encoding. Nat Neurosci, 5:1339-44.

10. Reynolds JR, Donaldson DI, Wagner AD, Braver TS. (2004) Item- and task-level processes in the left inferior prefrontal cortex: positive and negative correlates of encoding. Neuroimage, Vol. 21:1472-83.

11. Schacter DL, Wagner AD. (1999) Medial temporal lobe activations in fMRI and PET studies of episodic encoding and retrieval. Hippocampus, Vol. 9:7-24. 
12. Uttal WR. (2001). The new phrenology: The limits of localizing cognitive processes in the brain. A Bradford book, MIT Press.

13. Visscher KM, Miezin FM, Kelly JE, Buckner RL, Donaldson DI, McAvoy MP, Bhalodia VM, Petersen SE. (2003). Mixed block/event-related designs can correctly separate transient and sustained activity in fMRI. NeuroImage, Vol 19: 1694-1708.

14. Velanova K, Jacoby LL, Wheeler ME, McAvoy MP, Petersen SE, Buckner RL. (2003) Functional-anatomic correlates of sustained and transient processing components engaged during controlled retrieval. J Neurosci, Vol. 23:8460-70.

15. Wagner AD, Schacter DL, Rotte M, Koutstaal W, Maril A, Dale AM, Rosen BR, Buckner RL. (1998) Building memories: remembering and forgetting of verbal experiences as predicted by brain activity. Science, Vol. 21:1188-91. 


\section{FIGURE 1:}

Different experimental paradigm designs can be used to dissociate, or parse, brain activity according to the functional role that the region plays in supporting behaviour. Rather than simply look at "where" in the brain is active during the performance of a task, mixed designs begin to allow neuroimaging to provide additional information about "what" the different regions are doing. The TOP PANEL illustrates the idea that neuroimaging provides a measure of all the processing that is going on within the brain during performance of a task. To ask 'what' rather than just 'where' questions, the critical issue is how to separate out (parse) this activity into meaningful psychological/functional units. Pragmatically, the most straightforward and tractable divisions (boundaries) between levels are defined experimentally, in terms of tasks, trials/stimuli and sub-trial events. The BOTTOM PANEL illustrates the different types of signals that different methods measure. Blocked designs reveal all of the regions active during a task (1), but the activity could reflect numerous underlying patterns of signal change. Event-related designs isolate regions that exhibit a transient response to individual trials (2), whereas mixed designs can also identify regions that exhibit a sustained response that is independent of a given trial, but nonetheless supports performance at the broader taskrelated level (3).

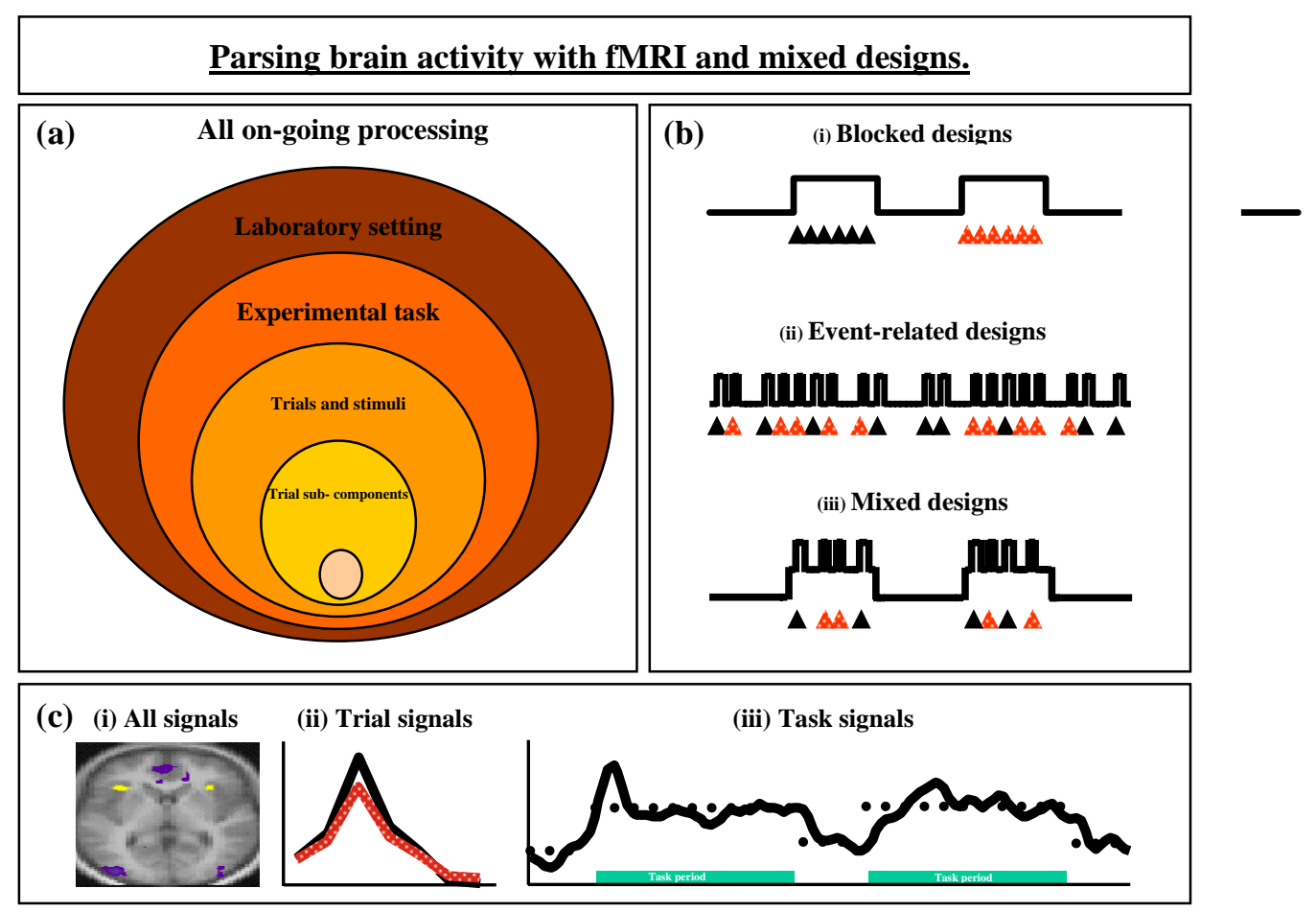

